\title{
Real Time Observation of Nanomaterials in Energy Harvesting and Li-ion Battery Systems
}

\author{
R.S. Yassar,* H. Ghassemi,* A. Asthana,* M. Au, ** and Y.K. Yap*** \\ * Mechanical Engineering-Engineering Mechanic Department, Michigan Technological University, \\ Houghton, MI 49931 \\ ** Savannah River National Laboratory, Aiken, SC 29808 \\ *** Physics Department, Michigan Technological University, Houghton, MI 49931
}

In energy systems such as Li-ion battery and energy harvesting devices, materials may experience a combination of strain, electrical, electrochemical, and thermal fields. This paper summarizes the application of in situ microscopy on the effect of such extreme environments on nanotubes and nanowires. Boron nitride $(\mathrm{BN}), \mathrm{TiO}_{2}, \mathrm{ZnO}$, and $\mathrm{Si}$ nanowires were studied under electrochemical coupling (Li-ion battery) and mechano-electrical coupling (piezoelectric harvesting devices). All the experimental measurements were conducted inside the chamber of a transmission electron microscope (TEM) using an in-situ scanning tunneling microcopy (STM) holder.

This study reports for the first time the buckling mechanism in multi-walled BNNTs upon severe mechanical deformation [1]. High-resolution TEM images revealed that bent BNNTs form multiple rippling upon buckling [2]. The rippling wavelength was quantified in terms of the outer diameter and thickness of nanotubes (Fig.1). BNNTs showed larger rippling wavelength compared to that of CNTs with the same number of walls. This difference was explained by the tendency of BN structures to reduce the number of thermodynamically unfavorable B-B and N-N bonds at the sharp corners in the rippling regions. BNNTs structure also exhibited higher fracture strain compared to their counterpart.

$\mathrm{TiO}_{2}$ and $\mathrm{Si}$ nanotubes are promising materials for Lithium-ion batteries. We studied the in-situ observation of lithiation and delithiation in $\mathrm{Si}$ and $\mathrm{TiO}_{2}$ nanotubes. Ionic liquids (ILs) were entered into the chamber of TEM successfully allowing the electrochemical reactions to be observed in real time (Fig. 2). The intercalation of crystalline anatase and amorphous $\mathrm{TiO}_{2}$ was studied and their fracture events were monitored.

Energy harvesting from individual nanowires and nanotubes has become an interesting area of research and there are uncertainties associated with the mechanism of such energy signals. Here we have used in-situ electron microscopy to observe the piezoelectric current that is being generated by mechanical deformation of a single $\mathrm{ZnO}$ nanowire (Fig. 3). This energy was quantified as a function of $\mathrm{ZnO}$ nanowire size scale and the effect of load mode on the magnitude of the generated current signal was studied.

References:

[1] H.M.S. Ghassemi et al., Nanotechnology, 22 (2011), 115702-115708

[2] H.M.S. Ghassemi, et al., Journal of Applied Physics, 108 (2010), 024314-4 

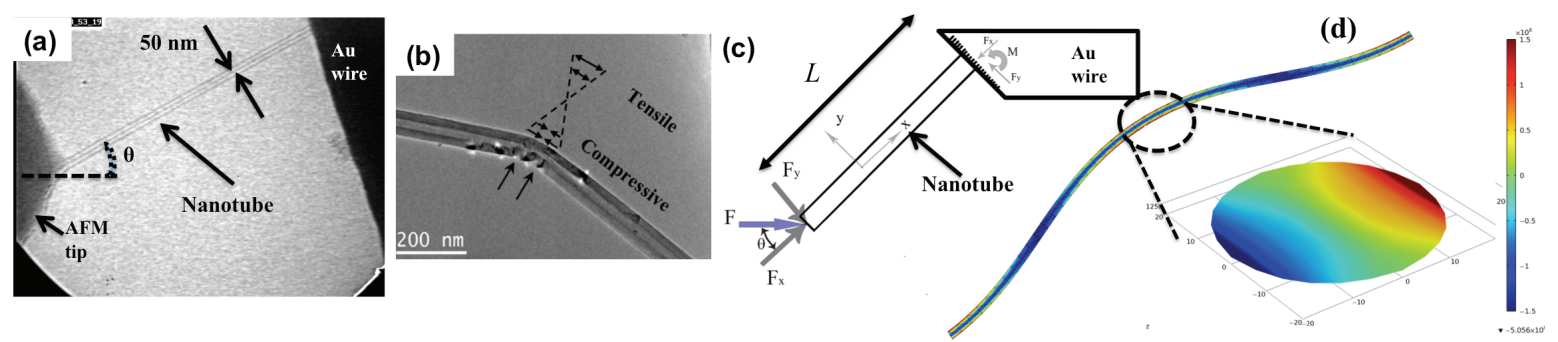

FIG 1. In-situ nanomechanical testing of a single BN nanotube is shown. (a) the nanotube is placed between AFM tip and Au wire, (b) the nanotube is bent, tensile and compressive stresses form on the surface of nanotube. Several ripplings form on the compressive side of nanotube (shown by arrows). (c) the free-body diagram of forces acting on the nanotube under the stress state shown in (a). (d) Finite element modeling of the bending process of the nanotube in (a) is shown.
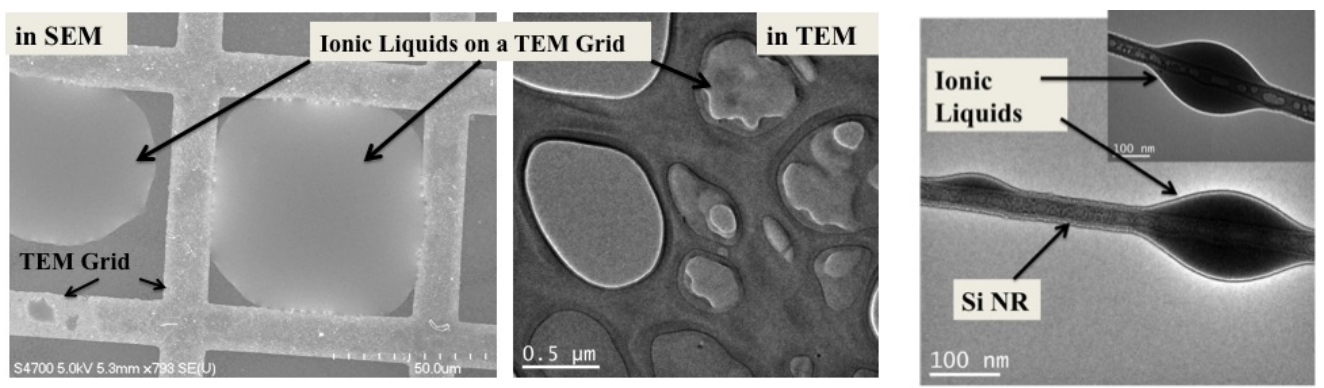

FIG 2. (left) EMIM TFSI ionic liquids were entered in the chamber of SEM and TEM. The round shape of ionic liquids indicates that they are in liquid state under the vacuum conditions and no evaporation or degassing occurred. (right) A nanorod that is in contact with ionic liquids is shown. The ionic liquids form as droplets on the surface of nanorods (marked with arrows).
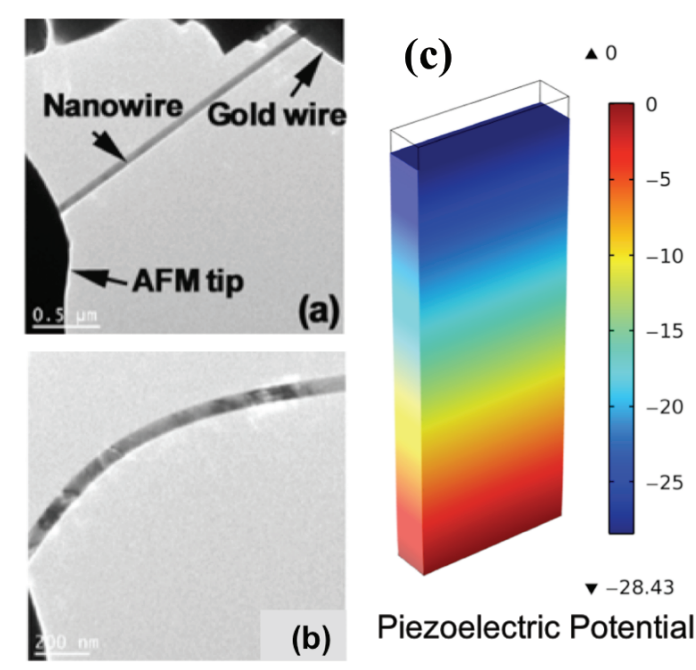

(b)

Piezoelectric Potential

FIG 3. (a-b) In-situ energy harvesting from a single $\mathrm{ZnO}$ nanowire. (c) The piezoelectric potential developed in $\mathrm{ZnO}$ under uniaxial stressing. 